\title{
Pritid
}

Physica A $216(1995) 185-194$

\section{On the number of degrees of freedom in Schwinger's quantum kinematics}

\author{
Augusto Cesar Lobo, M.C. Nemes \\ Universidade Federal de Minas Gerais, Instituto de Ciências Exatas, Depto de Fisica, \\ Caixa Postal 702 CEP 31270-901, Belo Horizonte, M.G., Brazil
}

Received 26 September 1994

\begin{abstract}
Some algebraic properties of Schwinger's quantum kinematical phase space theory are presented. These properties lead to a definition of the maximum number of degrees of freedom of an arbitrary finite dimensional quantum system which is different from the one originally proposed by Schwinger.
\end{abstract}

\section{Introduction}

The search for classical structures in quantum mechanics has been a challenging theoretical problem since the foundations of quantum theory.

After the establishment of the universality of chaotic behaviour and the subsequent quest for chaotic properties in quantum mechanics [1] this issue has received a new income of interest.

One of the earliest steps in this direction was that of the Wey-Wigner formalism [2]. It is essentially a kinematical approach that relates quantum mechanical particle observables to classical ones. The quantum state space is infinite dimensional and it is spanned by the continuous indexed basis of position and momentum particle eigenstates.

Schwinger introduced, some time ago, a quantum kinematical construction for finite dimensional spaces that is analogous to the infinite dimensional one [3]. In the past few years, there has been a renewal of interest in the concept of these finite phase spaces and it has been proposed as a natural route to generalize the Wey-Wigner formalism to finite dimensional systems [4]. Also, some interesting algebraic properties have been studied, which bear a very close analogy to those of continuous classical phase spaces [5].

In the case of infinite dimensional quantum systems, the problem of defining the number of degrees of freedom is rather straightforward. The problem is not so simple 
for finite dimensional quantum systems. Clarifying this point is the main purpose of this work. We show that in the latter case, the tensor product of two finite dimensional quantum spaces can be reduced to a single degree of freedom vector space if the dimensions of the original spaces are relatively prime. Such a result will be obtained as a natural consequence of some elementary facts of number theory.

The paper is organized in the following way:

In Section 2, we review Schwinger's theory as a direct discrete analogy of the continuum case, presenting the notations and form to be used later.

In Section 3 we derive the necessary algebraic properties and their consequences which are the main result of this paper. Such properties lead to a definition of the maximum number of degrees of freedom for an arbitrary finite dimensional quantum system that is not quite the same one proposed originally by Schwinger. In Section 4 we present some closing remarks and open problems.

\section{The finite phase space}

\subsection{The continuum case}

Let $W$ be the infinite dimensional quantum state space with position and momentum states $|q\rangle$ and $|p\rangle$ of a non-relativistic single particle motion in one dimension. The kets $|q\rangle$ and $|p\rangle$ stand for eigenstates of the usual pair of conjugate operators $(\hat{Q}, \hat{P})$ defined by the well known properties (we use from now on $\hbar=1$ )

"Commutation relations" $\left\{\begin{array}{l}{[\hat{Q}, \hat{P}]=\mathrm{i} \hat{1},} \\ {[\hat{Q}, \hat{Q}]=[\hat{P}, \hat{P}]=0,}\end{array}\right.$

"Completeness relation" $\left\{\int \mathrm{d} p|p\rangle\left\langle p\left|=\int \mathrm{d} q\right| q\right\rangle\langle q|=\hat{1}\right.$,

where $\hat{1}$ is the unity operator on $W$.

"Normalization conditions" $\left\{\begin{array}{l}\left\langle p \mid p^{\prime}\right\rangle=\delta\left(p-p^{\prime}\right), \\ \left\langle q \mid q^{\prime}\right\rangle=\delta\left(q-q^{\prime}\right),\end{array}\right.$

"Overlap equation" $\left\{\langle q \mid p\rangle=(2 \pi)^{-1 / 2} \mathrm{e}^{\mathrm{iqp}}\right.$.

The unitary translation operators on both position and momentum spaces are

$$
\hat{V}_{q}(\eta)=\mathrm{e}^{\mathrm{i} \eta \hat{P}}, \quad \hat{U}_{p}(\varepsilon)=\mathrm{e}^{\mathrm{i} \varepsilon \hat{Q}} .
$$

The above operators obey the usual properties

$$
\begin{array}{ll}
\hat{U}_{p}(\varepsilon)|p\rangle=|p+\varepsilon\rangle, & \hat{U}_{p}(\varepsilon)|q\rangle=\mathrm{e}^{\mathrm{i} \varepsilon q}|q\rangle \\
\hat{V}_{p}(\eta)|q\rangle=|q+\eta\rangle, & \hat{V}_{q}(\eta)|p\rangle=\mathrm{e}^{\mathrm{i} \eta p}|p\rangle
\end{array}
$$

which imply the following equations:

$$
\hat{U}_{p}(\varepsilon) \hat{V}_{q}(\eta)|p\rangle=\hat{U}_{p}(\varepsilon)|p\rangle \mathrm{e}^{\mathrm{i} \eta p}=|p+\varepsilon\rangle \mathrm{e}^{\mathrm{i} \eta p}
$$


and

$$
\hat{V}_{q}(\eta) \hat{U}_{p}(\varepsilon)|p\rangle=\hat{V}_{p}(\eta)|p+\varepsilon\rangle=\mathrm{e}^{\mathrm{i} \eta(p+\varepsilon)}|p+\varepsilon\rangle .
$$

By comparing (2.3a) and (2.3b) we get

$$
\hat{V}_{q}(\eta) \hat{U}_{p}(\varepsilon)=\mathrm{e}^{\mathrm{i} \eta \varepsilon} \hat{U}_{p}(\varepsilon) \hat{V}_{p}(\eta)
$$

This equation will be important, later on, when it will be compared with its finite dimensional version.

We could have started, in a rather more economical way, with the position space and the position operator and introduced the momentum operator as the hermitian generator of unitary translations in $q$-space. There is an evident symmetry here. We could otherwise have started with $p$-space and introduced the position operator as a translation generator in momentum space [6]. In any way all the properties listed above would follow immediately.

This procedure is Schwinger's starting point for the finite case as we recall in Section 2.2 below. Let us consider now two different degrees of freedom. We define then two spaces $W_{1}, W_{2}$ each one with a correspondent pair of conjugate operators $\left(\hat{Q}_{1}, \hat{P}_{1}\right),\left(\hat{Q}_{2}, \hat{P}_{2}\right)$. The total space is the tensor product of the individual spaces:

$$
W=W_{1} \otimes W_{2} \text {. }
$$

Each set of operators acts only on their own space. That is:

$$
\left[\hat{Q}_{1}, \hat{P}_{1}\right]=\left[\hat{Q}_{2}, \hat{P}_{2}\right]=\mathrm{i} \hat{I}
$$

and

$$
\left[\hat{Q}_{1}, \hat{Q}_{2}\right]=\left[\hat{P}_{1}, \hat{P}_{2}\right]=\left[\hat{Q}_{1}, \hat{P}_{2}\right]=\left[\hat{Q}_{2}, \hat{P}_{1}\right]=0
$$

The total position and momentum states are

$$
|\boldsymbol{p}\rangle=\left|p^{(1)}\right\rangle \otimes\left|p^{(2)}\right\rangle \equiv\left|p_{1}, p_{2}\right\rangle, \quad|\boldsymbol{q}\rangle=\left|q^{1}\right\rangle \otimes\left|q^{2}\right\rangle \equiv\left|q^{1}, q^{2}\right\rangle .
$$

The total position and momentum states are

$$
\left\{\begin{array} { l } 
{ \hat { V } _ { q ^ { 1 } } ( \eta ^ { 1 } ) = \mathrm { e } ^ { \mathrm { i } \hat { P } _ { 1 } \eta ^ { 1 } } } \\
{ \hat { U } _ { p _ { 1 } } ( \varepsilon _ { 1 } ) = \mathrm { e } ^ { \mathrm { i } \varepsilon _ { 1 } \hat { Q } ^ { 1 } } }
\end{array} \text { and } \quad \left\{\begin{array}{l}
\hat{V}_{q^{2}}\left(\eta^{2}\right)=\mathrm{e}^{\mathrm{i} \hat{P}_{2} \eta^{2}} \\
\hat{U}_{p_{2}}\left(\varepsilon_{2}\right)=\mathrm{e}^{\mathrm{i} \varepsilon_{2} \hat{Q}^{2}}
\end{array}\right.\right.
$$

Of course, each pair satisfies an equation similar to (2.4).

For a given vector $\boldsymbol{\eta}=\left(\begin{array}{l}\eta^{1} \\ \eta^{2}\end{array}\right)$, the translation operator $\hat{V}_{q}(\eta)=\hat{V}_{q^{1}}\left(\eta^{1}\right) \otimes \hat{V}_{q^{2}}\left(\eta^{2}\right)$ acts on $W$ as

$$
\hat{V}_{q}(\boldsymbol{\eta})|\boldsymbol{q}\rangle=|\boldsymbol{q}-\boldsymbol{\eta}\rangle \text {. }
$$

Thus, the set of points of the $q^{1}-q^{2}$ plane obtained, as we apply the $\hat{V}_{q}(\boldsymbol{\eta})$ operator on the $|q\rangle$ state, ly of course, on a straight line. Suppose, otherwise, one could find (for a certain $\boldsymbol{\eta}$ ) an operator $\hat{V}_{\boldsymbol{q}}(\boldsymbol{\eta})$ that applied on the $|\boldsymbol{q}\rangle$ state should cover the entire $q^{1}-q^{2}$ plane. Obviously, this is impossible for the infinite-dimensional case. But that is not so for finite spaces when the dimensions of each space are relatively prime, as we 
shall see. In this case it is impossible to sustain the concept that the product space has two degrees of freedom.

\subsection{Schwinger's quantum kinematics}

Let $W$ be a $N$-dimensional state space generated by an orthonormalized base $\left\{\left|\mu_{k}\right\rangle\right\}, k=1,2, \ldots, N$, so that $\left\langle\mu^{k} \mid \mu_{l}\right\rangle=\delta_{l}^{k}$. The upper indices are for dual base vectors and the sum convention for repeated lower and upper indices is used throughout, unless explicitly stated. The completeness of the $\left\{\left|\mu_{k}\right\rangle\right\}$ basis can now be written as: $\left|\mu_{k}\right\rangle\left\langle\mu^{k}\right|=\hat{1}$.

Next, an unitary operator $\hat{V}$ is defined according to cyclic permutation over $\left\{\left|\mu_{k}\right\rangle\right\}$ as follows:

$$
\begin{aligned}
& \hat{V}\left|\mu_{k}\right\rangle=\left|\mu_{k-1}\right\rangle, \quad k=1,2, \ldots, N \\
& \text { with }\left|\mu_{0}\right\rangle=\left|\mu_{N}\right\rangle \text { "periodic boundary condition" }
\end{aligned}
$$

From (2.7) one immediately gets $\hat{V}^{N}=\hat{1}$. Suppose now that $\left|v_{k}\right\rangle$ is a normalized eigenket of $\hat{V}$ with eigenvalue $v_{k}$. Then

$$
\hat{V}^{N}\left|v_{k}\right\rangle=\left(v_{k}\right)^{N}\left|v_{k}\right\rangle=\left|v_{k}\right\rangle . \quad \text { So }\left(v_{k}\right)^{N}=1 \quad \text { and } \quad v_{k}=\mathrm{e}^{(2 \pi \mathrm{i} / N) k} .
$$

That is, there are $N$ distinct eigenvalues given by the $N$ th squares of unity and the set of $N\left\{v_{k}\right\}$ states is also an orthonormalized basis $\left\{\left|v_{k}\right\rangle\right\}, k=1,2, \ldots, N$. Let us now consider the operator $\hat{P}\left(v_{k}\right)=(1 / N) v_{-j}^{k} \hat{V}^{j}$.

In this equation the $k$ index has a double function: It is the power of the $v_{-j}$ phase but it is also the $k$ index of the "matrix element" $v_{-j}^{k}$, where $-j$ is the same as $N-j$. It is easy to verify the important relation:

$$
\frac{1}{N} v_{-j}^{k} v_{l}^{j}=\delta_{l}^{k}
$$

so that: $\hat{P}\left(v_{k}\right)\left|v_{l}\right\rangle=\delta_{l}^{k}\left|v_{l}\right\rangle$. This means that $\hat{P}\left(v_{k}\right)$ "projects states in the $\left|v_{k}\right\rangle$ direction". So we can write: $\hat{P}\left(v_{k}\right)=\left|v_{k}\right\rangle\left\langle v^{k}\right|$ "no sum over $k$ !"

By applying $\hat{P}\left(v_{k}\right)$ over $\left|\mu_{N}\right\rangle$ we get $\hat{P}\left(v_{k}\right)\left|\mu_{N}\right\rangle=\left|v_{k}\right\rangle\left\langle v^{k} \mid \mu_{N}\right\rangle$ (notice that here no sum over $k$ is implied!) $=(1 / N) v_{-j}^{k} \hat{V}^{j}\left|\mu_{N}\right\rangle=(1 / N)\left|\mu_{N-j}\right\rangle v_{k}^{-j}$, then

$$
\left\langle\mu^{N}\left|\hat{P}\left(v_{k}\right)\right| \mu_{N}\right\rangle=\left|\left\langle\mu^{N} \mid v_{k}\right\rangle\right|^{2}=\frac{1}{N} \delta_{N-j}^{N} v_{k}^{-j}=\frac{1}{N} \delta_{-j}^{0} v_{k}^{-j}=\frac{v_{k}^{0}}{N}=\frac{1}{N} .
$$

Making the following phase choice $\left\langle\mu^{N} \mid v_{k}\right\rangle=1 / \sqrt{N}$, we have then

$$
\left\langle\mu^{N}\left|\hat{\boldsymbol{V}}^{l}\right| v_{k}\right\rangle=\left\langle\mu^{l} \mid v_{k}\right\rangle=\frac{v_{k}^{l}}{\sqrt{N}} .
$$

We thus observe indeed that $v_{k}^{l}$ is a matrix element of some sort. 
A completely analogous procedure can be used to construct an unitary operator $\hat{U}$ that acts upon the $\left\{\left|v_{k}\right\rangle\right\}$ basis by the cyclic permutation:

$$
\hat{U}\left|v_{k}\right\rangle=\left|v_{k+1}\right\rangle \text {. }
$$

Similarly one can verify that $\hat{U}$ has the same spectrum of $\hat{V}$, that is: $\mu_{k}=v_{k}=\mathrm{e}^{(2 \pi i / N) k}$ and, what is most important, the eigenstates of $\hat{U}$ are the original $\left|\mu_{k}\right\rangle$ base states! In fact $\left.\hat{U}\left|\mu_{j}\right\rangle=\hat{U}\left|\mu_{k}\right\rangle\left\langle v^{k} \mid \mu_{j}\right\rangle=\hat{U}\left|v_{k}\right\rangle v_{j}^{-k} / \sqrt{N}=(1 / \sqrt{N})\left|v_{k+1}\right\rangle v_{j}^{-k}=(1 / \sqrt{N})\left|\mu_{l}\right|\right\rangle$ $\times\left\langle\mu_{l} \mid v_{k+1}\right\rangle v_{j}^{-k}=(1 / N)\left|\mu_{l}\right\rangle v_{k+1}^{l} v_{j}^{-k}=(1 / N) v_{l} v_{k}^{l} v_{j}^{-k}\left|\mu_{l}\right\rangle=(1 / N) v_{l} N \delta_{j}^{l}\left|\mu_{l}\right\rangle$ $=v_{j}\left|\mu_{j}\right\rangle$ where we have used the defining equations for $\hat{U}$ and $\hat{V}$ and also Eqs. (2.9) and (2.10).

Another very important property is the commutation relation between different powers of $\hat{U}$ and $\hat{V}$ :

$$
\hat{U}^{l} \hat{V}^{k}\left|v_{m}\right\rangle=v_{m}^{k} \hat{U}^{l}\left|v_{m}\right\rangle=v_{m}^{k}\left|v_{m+l}\right\rangle
$$

and

$$
\hat{V}^{k} \hat{U}^{l}\left|v_{m}\right\rangle=\hat{V}^{k}\left|v_{m+l}\right\rangle=v_{m+l}^{k}\left|v_{m+l}\right\rangle \text {. }
$$

Comparing (2.12a) and $(2.12 b)$ one obtains

$$
\hat{V}^{k} \hat{U}^{l}=v^{k l} \hat{U}^{l} \hat{V}^{k}
$$

This equation is the finite dimensional analogous of Eq. (2.4). The $\left|\mu_{j}\right\rangle$ states correspond to the "position" $|q\rangle$ kets, while the $\left|v_{j}\right\rangle$ states are the "momentum" $|p\rangle$ kets. The $\hat{U}$ and $\hat{V}$ operators implement unitary "translations" of momentum and position states.

The finite "index set" of $N$ elements $k=1,2, \ldots, N$ can be understood as the momentum and position eigenvalues. We denote this set as $Z_{N}$ for the reason to be explained in the next section. In this way, we can define as the phase space, the finite collection of $N^{2}$ elements given, by the cartesian product $Z_{N} \times Z_{N}$. This is to be compared to the continuum case where phase space is the set of pairs $(p, q) \in \mathbb{R}^{2}$.

\section{Algebraic properties of finite phase spaces}

The main reference for this section is [7].

\section{1. $\bmod N$ algebra}

We start by recalling some definitions and properties of elementary algebra:

(1) $A$ set $A$ is a ring if there are two operations defined on it: The addition operation (denoted by the "sum" symbol " + ") by which it is an abelian group with a "zero element" 0 as the identity. The second operation is the multiplication, denoted by the symbol ".", together with the associative property and the distributive property. 
(2) If besides this structure, there is also an identity element for a commutative multiplication, it is said that $\mathrm{A}$ is a commutative ring with an identity element.

It is easy to verify that the set of integers $Z$ is a commutative ring with an identity element. An equivalence relation on $Z$ can be established for each $N \in Z$ in the following manner: for $a, b \in Z$ we say that $a$ is equivalent to $b(\bmod N)$ if $N$ divides there difference. That is:

$$
a \sim b \Leftrightarrow N /(a-b)
$$

where $x / y$ means " $x$ divides $y$ ".

This equivalence relation partitions $Z$ in $N$ disjoint sub-sets each one representing an equivalence class.

The first $N$ positive integers $\{0,1, \ldots, N-1\}$ represents, each one, a different class. We call $Z_{N}=\left\{[0]_{N},[1]_{N}, \ldots,[N-1]_{N}\right\}$ the set of such equivalent classes and denote by in the map, that associates to every integer $K$, the class $[K]_{N}$ to which it belongs. That is

$$
i_{N} Z \rightarrow Z_{N}, \quad K \mapsto[K]_{N}
$$

It can be shown that $Z_{N}$ is also a commutative ring with an identity element and that $i_{N}$ is a ring homomorfism, that is, $i_{N}$ "preserves" the ring structure of $Z$.

Let $\phi_{N}$ be the sub-set of $\left\{[1]_{N},[2]_{N}, \ldots,[K]_{N}, \ldots,[N-1]_{N}\right\}$ where $K$ is relative prime to $N$. The number of elements of this sub-set is denoted by $\phi(N)$ and is known as Euler's totient function. It can be shown that the set $\phi_{N}$ together with the multiplication operation inherited from $Z_{N}$ is a commutative group. In this way let us write

$$
\phi_{N}=\left\{\left[a_{1}\right]_{N},\left[a_{2}\right]_{N}, \ldots,\left[a_{\phi_{i N},}\right]_{N}\right\}
$$

and define as $[b]_{N}$ the product of all these elements:

$$
[b]_{N}=\left[a_{1}\right]_{N} \cdot\left[a_{2}\right]_{N} \cdots\left[a_{\phi_{(N)}}\right]_{N} .
$$

Let us multiply now each element by a same $[a]_{N} \in \phi_{N}$, obtaining, of course, a permutation of these elements (because $\phi_{N}$ is a group). Thus: $\left([a]_{N} \cdot\left[a_{1}\right]_{N}\right) \times$ $\left([a]_{N} \cdot\left[a_{2}\right]_{N}\right) \cdots\left([a]_{N} \cdot\left[a_{\phi_{(N)}}\right]_{N}\right)=[a]_{N}^{\phi(N)}=[b]_{N}=[b]_{N}$, which means that: $[a]_{N}^{\phi(N)}$ $=[1]_{N}$ or $[a]_{N}[a]_{N}^{\phi(N)-1}=[1]_{N}$.

Consequently we can find the inverse of any element of $\phi_{N}$ if the value of the totient function $\phi(N)$ is given as

$$
[a]_{N}^{-1}=[a]_{N}^{\phi(N)-1} .
$$

This result is the celebrated "Fermat-Euler theorem".

Notice that the finite set of position and momenta defined in the last section takes values in $Z_{N}$. Thus the finite phase space is the cartesian product of the $\operatorname{ring} Z_{N}$ by itself. 


\subsection{A system with two degrees of freedom}

Suppose now there are two spaces $W_{a}$ and $W_{b}$ with finite dimensions $N_{a}$ and $N_{b}$. Each space carries one degree of freedom in the sense of the formalism developed in Section 2.2. That is:

An unitary basis: $\left\{\left|\mu_{1}^{\left(N_{i}\right)}\right\rangle, \ldots,\left|\mu_{N_{i}}^{\left(N_{i}\right)}\right\rangle\right\}$ and $\left\{\left|v_{1}^{\left(N_{i}\right)}\right\rangle, \ldots,\left|v_{N_{i}}^{\left(N_{i}\right)}\right\rangle\right\}$ with: $\left\langle\mu^{\left(N_{i}\right) k} \mid v_{l}^{\left(N_{i}\right)}\right\rangle$ $=v_{l}^{\left(N_{i}\right) k}$ and $N_{i}=N_{a}$ or $N_{b}$ can be defined such that a pair of unitary translation operators $\left(\hat{U}^{\left(N_{i}\right)}, \hat{V}^{\left(N_{i}\right)}\right)$ can be constructed and obey the following relations: $\hat{V}^{\left(N_{i}\right)} \hat{U}^{\left(N_{i}\right)}=v_{1}^{\left(N_{i}\right)} \hat{U}^{\left(N_{i}\right)} \hat{V}^{\left(N_{i}\right)}$

$$
\begin{aligned}
& \text { and: }\left\{\begin{array}{l}
\hat{U}^{\left(N_{i}\right)}\left|\mu_{k}^{\left(N_{i}\right)}\right\rangle=\mu_{k}^{\left(N_{i}\right)}\left|\mu_{k}^{\left(N_{i}\right)}\right\rangle, \text { for the "position" eigenkets, } \\
\hat{V}^{\left(N_{i}\right)}\left|\mu_{k}^{\left(N_{i}\right)}\right\rangle=\left|\mu_{k}^{\left(N_{i}\right)}\right\rangle,
\end{array}\right. \\
& \text { and: }\left\{\begin{array}{l}
\hat{U}^{\left(N_{i}\right)}\left|v_{k}^{\left(N_{i}\right)}\right\rangle=\left|v_{k+1}^{\left(N_{i}\right)}\right\rangle, \\
\hat{V}^{\left(N_{i}\right)}\left|v_{k}^{\left(N_{i}\right)}\right\rangle=v_{k}^{\left(N_{i}\right)}\left|v_{k}^{\left(N_{i}\right)}\right\rangle,
\end{array}\right. \text { for the "momentum" eigenkets. }
\end{aligned}
$$

Let us consider the tensor produce space $W$ of $W_{a}$ and $W_{b}: W=W_{a} \otimes W_{b}$. The dimension of $W$ is clearly $N=N_{a} \cdot N_{b}$. One could think that $W$ should carry two degrees of freedom in a way similar to the continuum case. But that is not always true.

Suppose we succeed in defining a couple of basis for $W$ as

$$
\left|\mu_{t}^{(N)}\right\rangle=\left|\mu_{t_{a}}^{\left(N_{a}\right)}\right\rangle \otimes\left|\mu_{t_{b}}^{\left(N_{b}\right)}\right\rangle, \quad\left|v_{k}^{(N)}\right\rangle=\left|\mu_{k_{a}}^{\left(N_{a}\right)}\right\rangle \otimes\left|v_{k_{b}}^{\left(N_{b}\right)}\right\rangle
$$

with: $k, t \in Z_{N} ; k_{a}, t_{a} \in Z_{N_{u}} ; k_{b}, t_{b} \in Z_{N_{h}}$, and also a pair of unitary operators on $W$ :

$$
\hat{V}^{(N)}=\hat{V}^{\left(N_{a}\right)} \otimes \hat{V}^{\left(N_{b}\right)}, \quad \hat{U}^{(N)}=\hat{U}^{\left(N_{0}\right)} \otimes \hat{U}^{\left(N_{b}\right)}
$$

with: $r_{a}, s_{a} \in Z_{N_{a}}$ and $r_{b}, s_{b} \in Z_{N_{b}}$, such that they obey the correct commutation relation:

$$
\hat{V}^{(N)} \hat{U}^{(N)}=v_{1}^{(N)} \hat{U}^{(N)} \hat{V}^{(N)}
$$

Then $W$ has really only one true degree of freedom. Let us see for what conditions this

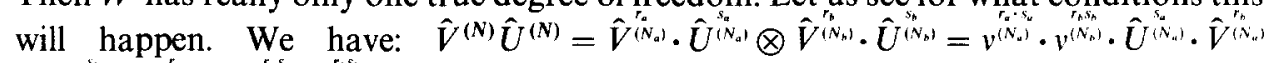
$\otimes \hat{U}^{\left(N_{+1}\right)} \cdot \hat{V}^{\left(N_{b}\right)}=v^{\left(N_{b}\right)} \cdot v^{\left(N_{b}\right)} \cdot \hat{U}^{(N)} \otimes \hat{V}^{(N)}$ and, using the identity:

$$
v_{k}^{\left(N_{a}\right)} \cdot v_{l}^{\left(N_{b}\right)}=v_{k N_{b}+l N_{a}}^{\left(N_{a} N_{b}\right)}
$$

together with (3.4), we get: $v_{r_{a} s_{a}}^{\left(N_{a}\right)} \cdot v_{r_{b} s_{b}}^{\left(N_{b}\right)}=v_{r_{a} s_{a} N_{b}+r_{b} s_{b} N_{a}}^{\left(N_{a} N_{b}\right)}=v_{1}^{(N)}$, which means that $\left[r_{a} s_{a} N_{b}+r_{b} s_{b} N_{a}\right]_{N}=[1]_{N}$.

The above diofantine equation [7] for $r_{a} s_{a} \in Z_{N_{a}}$ and $r_{b} s_{b} \in Z_{N_{b}}$ can be written as: $\left[1-r_{a} s_{a} N_{b}\right]_{N}=\left[r_{b} s_{b} N_{a}\right]_{N}$, so $N_{a} /\left(1-r_{a} s_{a} N_{b}\right)$, which means by definition that: $\left[r_{a} s_{a} N_{b}\right]_{N_{a}}=[1]_{N_{a}}$ (an equation in $Z_{N_{a}}$ ). In the same way we find a similar equation in $Z_{N_{b}}:\left[r_{b} s_{b} N_{a}\right]_{N_{b}}=[1]_{N_{b}}$, which can be solved uniquely as (using the Fermat-Euler theorem)

$$
\left[r_{a} s_{a}\right]_{N_{a}}=\left[N_{b}\right]_{N_{a}}^{-1}=\left[N_{b}\right]_{N_{a}}^{\phi\left(N_{a}\right)-1}
$$


and

$$
\left[r_{b} s_{b}\right]_{N_{b}}=\left[N_{b}\right]_{N_{b}}^{-1}=\left[N_{b}\right]_{N_{b}}^{\phi\left(N_{b}\right)-1}
$$

only if $N_{b} \in \phi_{N_{a}}$ and $N_{a} \in \phi_{N_{b}}$, that is, if $N_{a}$ and $N_{b}$ are relatively prime. We must also impose that

$$
\hat{V}^{(N)}\left|v_{k}^{(N)}\right\rangle=v_{k}^{(N)}\left|v_{k}^{(N)}\right\rangle=\hat{V}^{\left(N_{a}\right)} \otimes \hat{V}^{\left.r_{b}\right)}\left|v_{k_{b}}^{\left(N_{b}\right)}\right\rangle \otimes\left|v_{k_{h}}^{\left(N_{a}\right)}\right\rangle=v_{k_{a} r_{a}}^{\left(N_{b}\right)} \cdot v_{k_{b} r_{b}}^{\left(N_{b}\right)}\left|v_{k}^{(N)}\right\rangle
$$

and

$$
\hat{U}^{(N)}\left|\mu_{t}^{(N)}\right\rangle=\hat{V}_{k}^{(N)}\left|\mu_{t}^{(N)}\right\rangle=\hat{U}^{s_{a}\left(N_{a}\right)} \otimes \hat{U}^{\left(s_{b}\right)}\left|\mu_{t_{a}}^{\left(N_{a}\right)}\right\rangle \otimes\left|\mu_{t_{b}}^{\left(N_{a}\right)}\right\rangle=v_{s_{a} t_{a}}^{\left(N_{b}\right)} \cdot v_{s_{b} t_{b}}^{\left(N_{a}\right)}\left|\mu_{t}^{(N)}\right\rangle .
$$

Then from (3.5) we verify that

$$
[k]_{N}=\left[N_{b} k_{a} r_{a}+N_{a} k_{b} r_{b}\right]_{N}, \quad[t]_{N}=\left[N_{b} s_{a} t_{a}+N_{a} s_{b} t_{b}\right]_{N} .
$$

Solving these diofantine equations, as before, one has

$$
\left[k_{a}\right]_{N_{a}}=[k]_{N_{a}} \cdot\left[N_{b} r_{a}\right]_{N_{a}}^{-1}, \quad\left[t_{a}\right]_{N_{a}}=[t]_{N_{a}}\left[N_{b} s_{a}\right]_{N_{a}}^{-1}
$$

and

$$
\left[k_{b}\right]_{N_{b}}=[k]_{N_{b}} \cdot\left[N_{a} r_{b}\right]_{N_{b}}^{-1}, \quad\left[t_{b}\right]_{N_{b}}=[t]_{N_{b}}\left[N_{b} s_{b}\right]_{N_{b}}^{-1} .
$$

So we conclude that if $N_{a}$ and $N_{b}$ are relatively prime there is only one true degree of freedom associated to the total vector space $W=W_{a} \otimes W_{b}$.

Let us consider two examples:

For $N_{a}=2$ and $N_{b}=3$, using Eqs. (3.6) and (3.7) and choosing the "vector"

$$
\boldsymbol{r}=\left(\begin{array}{l}
{\left[r_{a}\right]_{2}} \\
{\left[r_{b}\right]_{3}}
\end{array}\right)=\left(\begin{array}{l}
{[1]_{2}} \\
{[1]_{3}}
\end{array}\right)
$$

we obtain uniquely:

$$
\boldsymbol{s}=\left(\begin{array}{c}
{\left[s_{a}\right]_{2}} \\
{\left[s_{b}\right]_{3}}
\end{array}\right)=\left(\begin{array}{l}
{[1]_{2}} \\
{[2]_{3}}
\end{array}\right), \quad \boldsymbol{t}=\left(\begin{array}{c}
{\left[t_{a}\right]_{2}} \\
{\left[t_{b}\right]_{3}}
\end{array}\right)=\left(\begin{array}{l}
{[t]_{2}} \\
{[t]_{3}}
\end{array}\right) \quad \text { and } \boldsymbol{k}=\left(\begin{array}{c}
{\left[k_{a}\right]_{2}} \\
{\left[k_{b}\right]_{3}}
\end{array}\right)=\left(\begin{array}{c}
{[k]_{N_{a}}} \\
{[2 k]_{N_{b}}}
\end{array}\right) \text {, }
$$

so that: $\hat{V}^{(6)}=\hat{V}^{(2)} \otimes \hat{V}^{(3)}$ and $\hat{U}^{(6)}=\hat{U}^{2} \otimes \hat{U}^{(3) 2}$.

The two "position" and "momentum" basis vectors are:

$$
\begin{aligned}
& \left|\mu_{0}^{(6)}\right\rangle=\left|\mu_{0}^{(2)}\right\rangle \otimes\left|\mu_{0}^{(3)}\right\rangle, \quad\left|v_{0}^{(6)}\right\rangle=\left|v_{0}^{(2)}\right\rangle \otimes\left|v_{0}^{(3)}\right\rangle, \\
& \left|\mu_{1}^{(6)}\right\rangle=\left|\mu_{1}^{(2)}\right\rangle \otimes\left|\mu_{1}^{(3)}\right\rangle, \quad\left|v_{1}^{(6)}\right\rangle=\left|v_{1}^{(2)}\right\rangle \otimes\left|v_{2}^{(3)}\right\rangle \text {, } \\
& \left|\mu_{2}^{(6)}\right\rangle=\left|\mu_{0}^{(2)}\right\rangle \otimes\left|\mu_{2}^{(3)}\right\rangle, \quad\left|v_{2}^{(6)}\right\rangle=\left|v_{0}^{(2)}\right\rangle \otimes\left|v_{1}^{(3)}\right\rangle \text {, } \\
& \left|\mu_{3}^{(6)}\right\rangle=\left|\mu_{1}^{(2)}\right\rangle \otimes\left|\mu_{0}^{(3)}\right\rangle, \quad\left|v_{3}^{(6)}\right\rangle=\left|v_{1}^{(2)}\right\rangle \otimes\left|v_{0}^{(3)}\right\rangle \text {, } \\
& \left|\mu_{4}^{(6)}\right\rangle=\left|\mu_{0}^{(2)}\right\rangle \otimes\left|\mu_{1}^{(3)}\right\rangle, \quad\left|v_{4}^{(6)}\right\rangle=\left|v_{0}^{(2)}\right\rangle \otimes\left|v_{2}^{(3)}\right\rangle \text {, } \\
& \left|\mu_{5}^{(6)}\right\rangle=\left|\mu_{1}^{(2)}\right\rangle \otimes\left|\mu_{2}^{(3)}\right\rangle, \quad\left|v_{5}^{(6)}\right\rangle=\left|v_{1}^{(4)}\right\rangle \otimes\left|v_{1}^{(3)}\right\rangle \text {. }
\end{aligned}
$$

By acting upon the "position" states with the $\hat{V}^{(2)} \otimes \hat{V}^{(3)}$ operator, one finds easily that it covers the whole position space. 
That is, we cover the entire set with only one "straight line"! The same happens of course to the momentum base states. So the six-dimensional state space can represent only one true degree of freedom. This is because $N_{a}=2$ is relatively prime to $N_{b}=3$.

Suppose now: $N_{a}=2$ and $N_{b}=2$.

In this case $N_{a}$ and $N_{b}$ are not relatively prime, so the above machinery does not work. But we can construct the four dimensional position product space:

$$
\mathrm{I}\left\{\begin{array} { l } 
{ | \mu _ { 0 } ^ { ( 2 ) } \rangle \otimes | \mu _ { 0 } ^ { ( 2 ) } \rangle , } \\
{ | \mu _ { 1 } ^ { ( 2 ) } \rangle \otimes | \mu _ { 1 } ^ { ( 2 ) } \rangle , }
\end{array} \quad \text { II } \left\{\begin{array}{l}
\left|\mu_{1}^{(2)}\right\rangle \otimes\left|\mu_{0}^{(2)}\right\rangle, \\
\left|\mu_{0}^{(2)}\right\rangle \otimes\left|\mu_{1}^{(2)}\right\rangle .
\end{array}\right.\right.
$$

The actions of the $\hat{V}^{(2)} \otimes \hat{V}^{(2)}$ operator on this basis splits it in two disjoint sets each one a "straight line" in position space parallel to the other. A similar procedure could be carried out for the momentum space.

So we can see the four dimensional quantum space either as a two degree of freedom system spanned by the above basis or as a one degree of freedom system with the $\left\{\left|\mu_{j}^{(4)}\right\rangle\right\},(j=0,1,2,3)$ basis defined in the usual way. But, in no way can the two degree of freedom space be reduced into a single degree of freedom system as in the preceding example.

This shows that the number of degrees of freedom of an arbitrary $N$-dimensional quantum space cannot be the number of prime factors of $N$, as stated by Schwinger. In fact, let $N$ be any integer, then we can factorize $N$ as

$$
N=P_{1}^{\alpha_{1}} \cdot P_{2}^{\alpha_{2}} \ldots P_{i}^{\alpha_{i}} \ldots P_{m}^{\alpha_{m}}, \quad i=1, \ldots, m \text { and } \alpha_{1}<\alpha_{2}<\cdots<\alpha_{m},
$$

where there are $m$ prime numbers $P_{i}$, each one, with power $x_{i}$.

By Schwinger's definition, the maximum number of degrees of freedom $f$ would be: $f=\alpha_{1}+\alpha_{2}+\ldots+\alpha_{m}$.

However, as we have just seen, the product of two relatively prime numbers can represent only one true degree of freedom, so we propose that

$$
f=\max \left\{\alpha_{1}, \alpha_{2}, \ldots, \alpha_{m}\right\}=\alpha_{m} .
$$

That is, the maximum number of degrees of freedom of a finite $N$-dimensional quantum space is the largest of the powers of the unique factorization of $N$ by prime numbers.

\section{Conclusion}

In the present contribution, Schwinger's concept of finite dimensional phase space has been explored in an algebraic context. We have addressed the question of the definition of the number of degrees of freedom in such context and proposed a definition which comes on naturally from the algebraic properties derived in Section 3.

A possible relation between this "finite classical structure" and the "continuous symplectic structure" of quantum mechanical projective spaces ("space of rays") must not be discarded $[8,9]$. 
In the last case, the number of degrees of freedom is half the dimension of projective space.

What possible connection could there be (or not) between this definition and the similar one for Schwinger's quantum kinematical finite phase space?

We hope that the present work may help to shed some light in this search for "classical structures" in finite dimensional quantum kinematics.

\section{References}

[1] P.V. Elyutin, The quantum chaos problem, Sov. Phys. Usp. 31 (7) (July 1988).

[2] S.R. de Groot and L.G. Suttorp, Foundations of Electrodynamics (North-Holland, Amsterdam, 1972) chap. VI and Appendix.

[3] J. Schwinger, Unitary Operator Bases, Proc. Nat. Acad. Sci. 46 (1960) 570.

[4] A Wigner-Function formulation of finite-state quantum mechanics, Annal. Phys. 176 (May, 1985).

[5] R. Aldrovandi and D. Galetti, On the structure of quantum phase space, J. Math. Phys. 31 (12) (December, 1990).

[6] J.J. Sakurai, Modern Quantum Mechanics (Benjamin/Cumming, 1985).

[7] K. Ireland and M. Rosen, A Classical Introduction to Modern Number Theory (Springer, 1982).

[8] J. Anandan, Geometric phase fo cyclic motions and the quantum state space metric, Phys. Lett. A 147 (1990) 3.

[9] V.I. Arnold, Mathematical Methods of Classical Mechanics (Springer, 1978) Appendix 3. 